\title{
Modelación Hidráulica 2D de Inundaciones en Regiones con Escasez de Datos. El Caso del Delta del Río Ranchería, Riohacha-Colombia
}

\author{
Jhonny I. Pérez, Jairo R. Escobar y Jose M. Fragozo \\ Grupo de Investigación GISA, Facultad de Ingeniería, Universidad de La Guajira, Km 5 Vía a Maicao, \\ Riohacha-Colombia (e-mail: jpemon@uniguajira.edu.co; jrescobar@uniguajira.edu.co; \\ jomfrago2012@gmail.com)
}

Recibido Dic. 29, 2017; Aceptado Mar. 7, 2018; Versión final Abr. 2, 2018, Publicado Ago. 2018

\begin{abstract}
Resumen
Se planteó una estrategia metodológica que solventa la escasez de datos para la modelación 2D de inundaciones en un delta. Digitalizando la elevación disponible en Google Earth ${ }^{\mathrm{TM}} \mathrm{e}$ integrada con secciones levantadas en el río y datos topográficos, se obtuvo una nube de puntos topográfica. Esta fue posteriormente interpolada a una red de triángulos irregular, y finalmente, la generación de un Modelo Digital del Terreno. Se simuló bidimensionalmente (IBER v2.3.2) un evento de inundación y una medida estructural (dique) para proteger una comunidad vulnerable. El desbordamiento se presentó solamente en el tramo final del cauce, por tanto, no influyó sobre el área urbana de la ciudad de Riohacha. El dique lograría evitar artificialmente las inundaciones en la zona protegida, pero aumentando el riesgo por inundación ante posibles fallas debido a crecidas de mayor magnitud. De acuerdo a los resultados, la metodología planteada es útil para el cálculo de áreas inundables en zonas deltaicas y apoyo a la toma decisiones en regiones con escases de datos.
\end{abstract}

\section{D Hydraulic Flood Modeling in Data-Scarce Regions The Case of Ranchería River Delta, Riohacha-Colombia}

\begin{abstract}
A methodological approach which overcomes the lack of data available for 2D hydrodynamic modeling in deltaic floodplains is proposed. By digitizing the elevation data available in Google Earth ${ }^{\mathrm{TM}}$ and integrating them with river cross sections data surveyed on the field, plus additional topographic data available, a topographic point cloud was obtained. This was later interpolated in a Triangulated Irregular Network, and finally, a Digital Terrain Model was generated. A two-dimensional simulation (with IBER v2.3.2) for a flood event together with a solution alternative including a structural measure (levee) to protect a vulnerable community were carried out. Overflow occurred only in the final stretch of the riverbed. Therefore, it did not influence the urban area of the city of Riohacha. The levee would artificially avoid the flooding on the target area for protection but increasing the flood risk due to possible failure that would occur when a swelling river occurs. Based on results, the proposed methodology is useful for determining flood prone areas in deltaic zones and well supports decision making in data-scarce regions.
\end{abstract}

Keywords: 2D modeling; IBER; flood; digital terrain model 


\section{INTRODUCCIÓN}

El Cambio Climático (CC) y sus impactos asociados son una nueva realidad para la humanidad, a largo plazo puede ser una amenaza u oportunidad. Sin embargo, las rápidas variaciones que se han producido desde mediados del siglo pasado, se deben en gran medida a las emisiones de gases de efecto invernadero (GEI) de origen antrópico (OMM, 2012). Los modelos de circulación climática global, proyectan eventos de temperatura y lluvias mucho más intensas que los observados actualmente (Trenberth, 2011). Sumado a lo anterior, el crecimiento acelerado de la población global junto a una mayor frecuencia de lluvias extremas, aumentarían el riesgo local de inundaciones (Kundzewicz et al., 2014). A escala continental, en los trópicos concretamente, los patrones de precipitación son dominados principalmente por el fenómeno natural ENOS (El Niño u Oscilación del Sur). De ENOS se proyecta un incremento en su intensidad, explicado probablemente en parte por la presión ejercida del CC (Cai et al., 2015). Los efectos negativos de ENOS en el contexto de Latinoamérica se evidencian con fuertes inundaciones (La Niña) y periodos de sequía (EI Niño) cada vez más prolongados e intensos, escenarios que pueden llegar a ser más desfavorables de seguir la tendencia actual que simulan los escenarios de emisiones GEI (Tedeschi y Collins, 2016). En el contexto de Colombia, La Niña, ha generado la mayor ola invernal de los últimos 50 años durante el periodo 2010-2011, produciendo desbordamientos en ríos e inundaciones pluviales que generaron significativas pérdidas económicas y vidas humanas. Esto produjo la creación de una política nacional de adaptación ante la alta vulnerabilidad del país frente al CC (Hoyos et al., 2013). La aplicación de técnicas numéricas avanzadas como la modelación hidráulica son necesarias antes de una intervención física ingenieril en el proceso de evaluación del riesgo de inundación (Apel et al., 2009; Teng et al., 2017). Estas técnicas ofrecen el potencial para la zonificación del riesgo y la planificación participativa de alternativas de solución contra inundaciones de manera adecuada (Bodoque et al., 2016).

En las últimas dos décadas, se han realizado importantes avances en el modelado de inundaciones mediante el uso de una nueva generación de modelos numéricos hidráulicos bidimensionales (2D) como alternativa más completa y precisa, en determinados casos, a la modelación unidimensional (1D). El incremento de la oferta de información topográfica en formato de Modelos Digitales de Elevación (MDE) derivados de sensores espaciales satelitales y aerotransportados, han empujado aún más el empleo de la modelación 2D (Yan et al., 2015). Los MDE son una de las principales entradas de datos de elevación tipo ráster para la modelación hidrodinámica 2D (Musa et al., 2015), en el cual para cada posición X,Y es contendida el valor de elevación $Z$ dentro la matriz de información, y es obtenida comúnmente a partir de plataformas satelitales $u$ aerotransportadas (Ruslin et al., 2014). Los MDE se clasifican dependiendo si consideran todos los elementos de la superficie (MDS) o solamente el suelo desnudo a nivel del terreno (MDT). Pero esta información a nivel de detalle y periodicidad de captura en regiones como Latinoamérica no es accesible fácilmente, disponiéndose de MDE gratuitos de baja resolución, lo cual representa una gran limitante al igual que muchos otros lugares del mundo en vías de desarrollo. Un MDT detallado representa fielmente la configuración del territorio, aunque requiere mayor gasto computacional (Apel et al., 2009), el desafío es encontrar el nivel de detalle óptimo y escala para una necesidad especifica de modelación (de Moel et al., 2015). La modelación 2D demanda altos requerimientos en datos hidrográficos, capacidades computacionales elevadas y especialmente de datos topográficos (MDT) que limitan su aplicación en regiones donde la producción y acceso a estos datos es escasa y costosa de adquirir (Shen et al., 2015).

En algunas regiones de Latinoamérica, la modelación hidráulica 2D ha sido limitada por la escasez de datos adecuados tanto topográficos como hidroclimáticos. Países como Colombia con amplia oferta hídrica y de potencial hidroeléctrico cuentan con registros hidrográficos continuos especialmente parte alta y media de las principales cuencas del país, (Bedoya y López-Lezama, 2015). Sin embargo, la densidad de estaciones meteorológicas, la frecuencia de registro, datos faltantes y calidad de la información, son limitantes para la modelación de inundaciones, cuando las variaciones ocurren en términos de horas e incluso minutos. En zonas urbanas, la falta de datos es aún mayor, las lluvias que caen sobre ellas se transforman en escorrentía (inundaciones pluviales), aunado al desbordamiento de ríos (inundaciones fluviales), fenómenos que suceden mayormente como procesos naturales, aumentando el riesgo conjunto de inundaciones en zonas periurbanas Apel et al. (2016). Muchas veces estos fenómenos son exacerbados por el hombre debido a su efecto sobre el territorio, es decir el inadecuado urbanismo y la expansión poblacional. Esta expansión ha llevado a las ciudades de lugares más o menos seguros a lugares definitivamente inseguros, aumentando el riesgo de inundación (Franco, 2010). Dado el problema local de las inundaciones, el proceso de evaluación de riesgo por inundación cuenta con una amplia oferta de software o modelos hidrodinámicos 2D pagos y/o libres para el cálculo de variables como profundidades, caudales y velocidades en cauce como planicie de inundación. Encontramos algunos comunes como el FLO-2D, DSS-WISE, TELEMAC, INFO Works, LISFLOOD-FP, Flood Modeller Pro entre otros más, oportunamente descritos con sus limitantes y ventajas en Teng et al. (2017). Por otro lado, existe cada vez una oferta creciente de modelos $2 \mathrm{D}$ que funcionan bajo software gratuitos (libres) que merecen especial atención dada su accesibilidad como es el caso de HEC RAS 2D e IBER 2D. Bladé et al. (2014) han desarrollado el código para la simulación hidrodinámica 2D dentro del software IBER, 
el cual ha tenido un uso considerable en el contexto iberoamericano para modelar inundaciones fluviales (Bonasia et al., 2017; Bodoque et al., 2016). El software de IBER cuenta con una interfaz en pre y post proceso muy sencilla e intuitiva. Su carácter gratuito lo convierte en una herramienta accesible para la gestión del riesgo de inundaciones al alcance de académicos y tomadores de decisiones en países lberoamericanos.

En Colombia, una de las zonas más sensibles y vulnerables frente a los efectos extremos del Niño y la Niña es el departamento de La Guajira. Los amplios contrastes biogeográficos entre zonas semiáridas con bosque seco en el norte y altos relieves con bosque de montaña en el sur, configuran en la región oscilaciones climáticas extremas de sequias e inundaciones. Esto hace imperativo el desarrollo de medidas de prevención, mitigación y acción local frente fenómenos globales como el CC. Como antecedente local de estudios locales de inundaciones, se tiene el proyecto "Adaptación Urbana Verde Frente a Inundaciones con el Soporte de la Modelación Matemática MODCEL en Riohacha, La Guajira-Colombia”, que en adelante abreviará como MODCEL, en el cual se plantearon alternativas de solución a inundaciones pluviales urbanas determinando previamente las cotas del agua en los límites entre la zona periurbana y el delta del río Ranchería (CREACUA, 2015; Nardini y Miguez, 2016). Una zona altamente impactada durante la ola invernal nacional de 2010-2011, fue el tramo final y principal del delta del río Ranchería conocido como El Riíto. Lo anterior y conociendo que gran parte de las aguas de la zona urbana drenan hacia el delta (sector El Rí́to), generó la motivación para desarrollar un estudio de modelación 2D con el fin de conocer la influencia de las crecidas del delta sobre el área urbana, en concreto el 18 de septiembre del 2011, máximo evento de lluvia e inundaciones pluviales en la ciudad. Este evento fue considerado también para la calibración en el proyecto MODCEL. Sin embargo, el uso directo de la topografía libre como MDT para la modelación 2D, no tenía la escala de detalle requerida y el detalle mostraba inconsistencias en representar el flujo superficial apropiadamente.

De acuerdo a lo anterior, se trazó como objetivo principal solventar la escasez de datos topográficos e hidrométricos para la modelación 2D de inundaciones, tomando el caso de estudio el delta del rio Ranchería (brazo El Riíto). Específicamente se propone: (i) una metodología para la generación de un MDT a escala apropiada para la modelación hidráulica (2D) en una zona deltaica; (ii) simular el evento de máxima precipitación (18/08/2011) usando el modelo IBER y (iii) simular el efecto de una medida de intervención estructural.

\section{METODOLOGÍA}

Se describe en forma separada diversos aspectos de la metodología empleada: área de estudio, herramienta computacional IBER 2D, propuesta metodológica del estudio y simulaciones efectuadas con IBER 2D.

\section{Área de estudio}

El delta del río Ranchería se encuentra ubicado al noreste de la ciudad de Riohacha (Caribe Colombiano). En la Figura 1 se muestra la localización general, el trazado del cauce principal del río Ranchería que se bifurca próximo a la línea de costa dando origen a los tres brazos que forman el delta, El Riíto (1), Calancala (2) y Santa Rita (3). El área de estudio de $12 \mathrm{~km}^{2}$, últimos $12 \mathrm{Km}$ del río y en línea recta $7,2 \mathrm{Km}$, iniciando en el puente de la vía que conduce a Maicao.

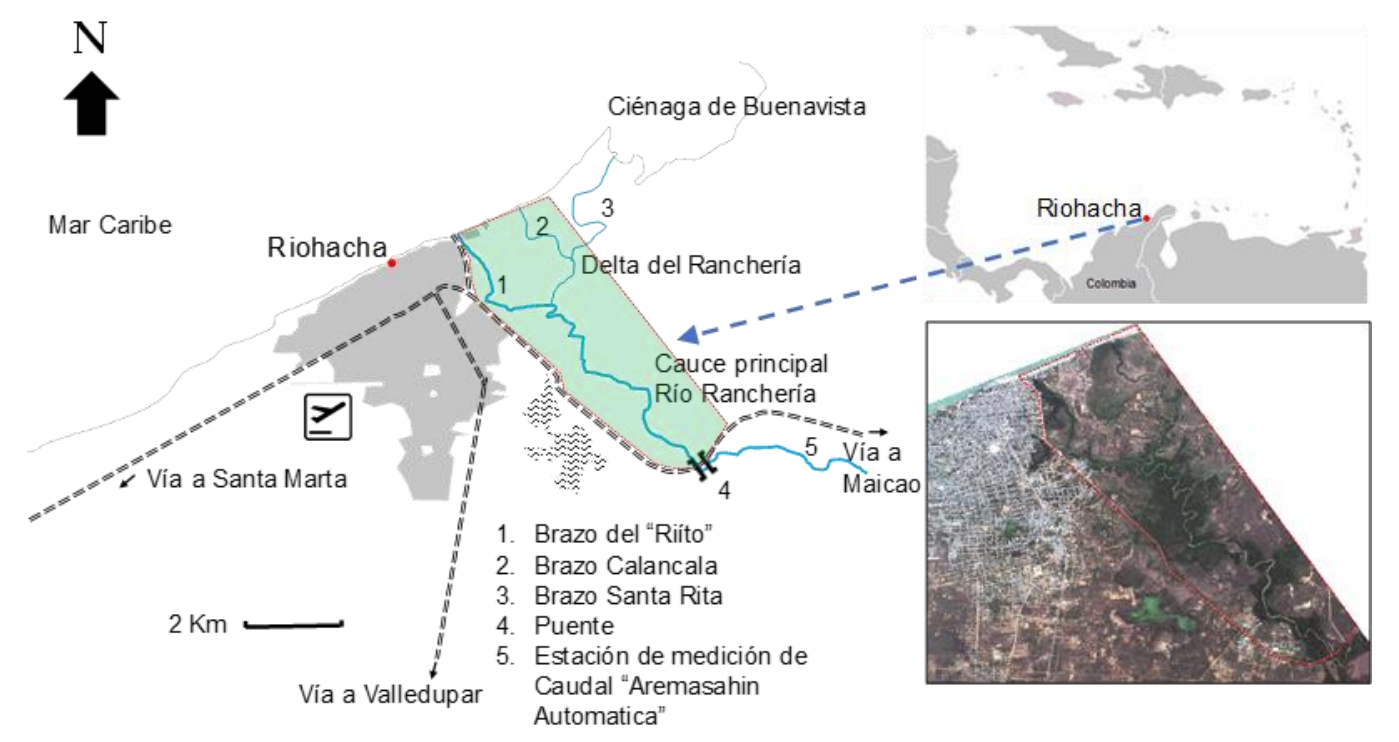

Fig. 1: Ubicación del delta del río Ranchería respecto a la zona urbana de Riohacha y desembocadura al Mar Caribe. 
El terreno es relativamente plano, suelos aluviales arcillosos y vegetación dominante arbustiva del bosque seco tropical y de manglar en cercanía a la costa. Según la autoridad meteorológica nacional (IDEAM), el régimen de lluvias de la región caribe es bimodal (en años con normalidad hidrológica). En Riohacha las lluvias totales anuales son de $541 \mathrm{~mm}$, siendo septiembre y octubre los meses más lluviosos. Parte de la zona del delta es destinada como área de preservación ambiental, aunque con asentamientos dispersos como es el caso de la comunidad sub-urbana de "Villa Fátima" ubicada entre los brazos (1) y (2) del río Ranchería, por tanto, expuesta periódicamente a inundaciones fluviales.

\section{Conceptualización de la modelación: La herramienta IBER 2D.}

El software IBER v2.3.2, fue usado para la simulación bidimensional bajo supuestos de flujo turbulento de lámina libre en régimen no-permanente aplicado en hidráulica fluvial. IBER 2D consta de diferentes módulos: turbulencia, transporte de sedimentos e hidrodinámica, constituyendo este último, la base de la herramienta empleando las ecuaciones de St. Venant 2D de aguas someras resueltas bajo el método de volúmenes finitos y sometidas al principio de conservación de la materia y de momento en las dos direcciones horizontales (Bladé et al., 2014). IBER 2D ha sido empleado en múltiples estudios de hidráulica aplicada: inundaciones fluviales (Bonasia et al., 2017), evaluación de medidas de restauración para pequeños cauces (Castillo et al., 2014), ruptura de represas (Álvarez et al., 2017) y transporte de material leñoso en ríos (Ruiz et al., 2015). Un input importante en IBER es la topografia a través de un MDT. IBER construye internamente la malla de cálculo, la cual representa la morfología del terreno en la hidrodinámica del modelo hidráulico a construir. En cada polígono de la malla se resuelven las ecuaciones hidrodinámicas una vez asignadas a la geometría, los valores de elevación del MDT y condiciones hidrodinámicas de contorno. En resumen, el flujo que emplea IBER 2D es: (i) generación de geometría de la zona: importación del MDT en formato ráster o ASCII; (ii) condiciones de contorno, definición de fronteras hidrodinámicas, contornos cerrados (tipo pared) y/o abiertos donde entra y sale el agua del dominio de cálculo; (iii) asignación de rugosidad, coeficiente de Manning de acuerdo a las coberturas del suelo; (iv) opciones generales de cálculo (tiempo de cálculo y parámetros del esquema numérico); (v) construcción interna de la malla de cálculo: estructurada que cada elemento tiene el mismo número de vecinos, o no estructurada (diferentes números de elementos); y (vi) ejecución de la simulación, análisis y validación.

En la Figura 2 se describe la metodología trazada en el estudio. La Figura 2a contiene la propuesta metodológica para generar el MDT en zonas de poca pendiente (deltas) y con escasez de datos topográficos. La Figura 2b muestra el esquema de simulación de alternativas con IBER 2D, Alternativa_0 que es el evento referencia bajo estudio (18/09/2011) y Alternativa_1 que consiste en simular un dique de protección.

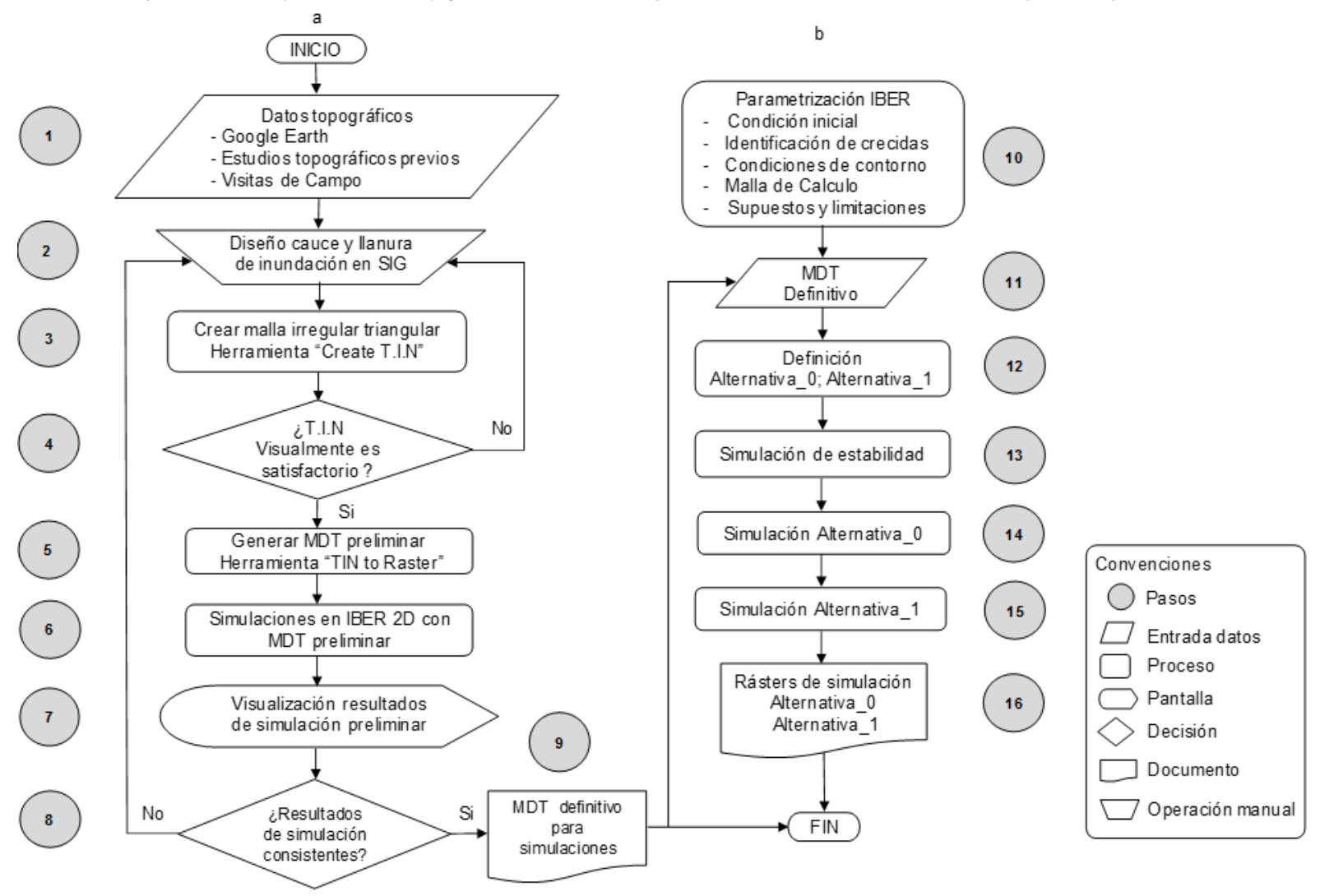

Fig. 2: Metodología trazada en el estudio. Propuesta metodológica para solventar la escasez de datos en la producción de un MDT (a) y esquema para la simulación de alternativas con IBER 2D (b). 


\section{Estrategia metodológica para solventar la escasez de datos}

A continuación, se describen los detalles del flujo descrito de la Figura 2a: (i) entrada de datos topográficos (paso 1); (ii) Diseño del cauce y llanura de inundación (paso 2); (iii) generación del MDT preliminar (pasos 3 a 5); iv) parametrización del software IBER 2D (paso 6); y (v) Obtención del MDT definitivo (pasos 7 a 9 ).

Entrada de datos topográficos. La fuente de datos de elevación de Google Earth ${ }^{\mathrm{TM}}$ de la Misión de Topografía por Radar de Transbordador Satelital (en inglés SRTM) y ASTER, fue la empleada por ser una de las pocas fuentes de información altimétrica de acceso libre y cubrimiento global para la determinación de datos puntales de ubicación y elevación en X, Y y Z (Hoffmann y Winde, 2010; Rusli et al., 2014). De esta fuente se parte en conjunto con la topografía convencional disponible, visitas de campo y el criterio de los investigadores, con el fin de capturar la configuración aproximada de la morfología del terreno de la planicie de inundación (paso 1). Los datos de elevación de Google Earth ${ }^{\mathrm{TM}}$ de altimetría (Z) solo se da en valores enteros de metros, así, la precisión es limitada con un uso restrictivo, impactando considerablemente el nivel de detalle del MDT a construir y la calidad final de los resultados de la simulación.

Diseño del cauce y llanura de inundación. Debido a la ausencia de datos altimétricos del terreno y batimétricos (equivalente subacuático de la altimetría), el cauce del río fue aproximado geométricamente a un canal tipo trapezoidal de configuración plana y de escasa pendiente propia de un delta (paso 2, Figura 3). El proceso ejecutado en este paso para llegar a un diseño integrado y consistente con la llanura de inundación consistió en: (i) digitalizar en un archivo de puntos shapefile en $X, Y$ para cada margen del rio en un Sistema de Información geográfico (SIG). 203 pares de puntos fueron necesarios para capturar la sinuosidad del río; (ii) aproximar el cauce a un canal trapezoidal procediendo a ejecutar el paso anterior, pero entre los pares de puntos ubicados en el cauce con el fin de establecer la base del fondo; (iii), la elevación Z para cada par de puntos con posición conocida $(X, Y)$ de las márgenes (figura 3a), se asignó mediante interpolación lineal una vez establecida la elevación al inicio y al final del cauce (desembocadura) que fue de 6 y 0 m.s.n.m respectivamente (supuesto de una pendiente constante de los márgenes); (iv) el procedimiento anterior fue aplicado para los puntos del fondo solo que trasladando verticalmente hacia abajo una profundidad promedio de 2,5 m aproximadamente en todo su recorrido, esto fue observado en las secciones levantadas en campo. En la Figura $3 b$ se detalla gráficamente el diseño de cauce y su aproximación trapezoidal. La nube de puntos $X, Y$ y $Z$ que discretiza el cauce idealizado del rio se guardó en un archivo de puntos ESRI shapefile (SHP) $2 D$ en un (SIG).

Dentro de la zona definida para la llanura de inundación, se asignaron puntos sobre el terreno de forma supervisada determinando la ubicación y elevación con Google Earth ${ }^{\mathrm{TM}}$. Se identificaron dentro del polígono puntos y áreas desprovistas de vegetación alta, viviendas, conocimiento de áreas inundables y demás puntos útiles para la construcción de un MDT que reflejase las características de la llanura de inundación con base al criterio y conocimiento empírico adquirido en terreno. Adicionalmente se integraron 23 puntos con elevaciones de información topográfica conocida de los box culvert existente en la vía a Maicao (CREACUA, 2015). Finalmente se generó un archivo shapefile de puntos que modeliza de forma aproximada la morfología de la llanura de inundación dentro del delta.
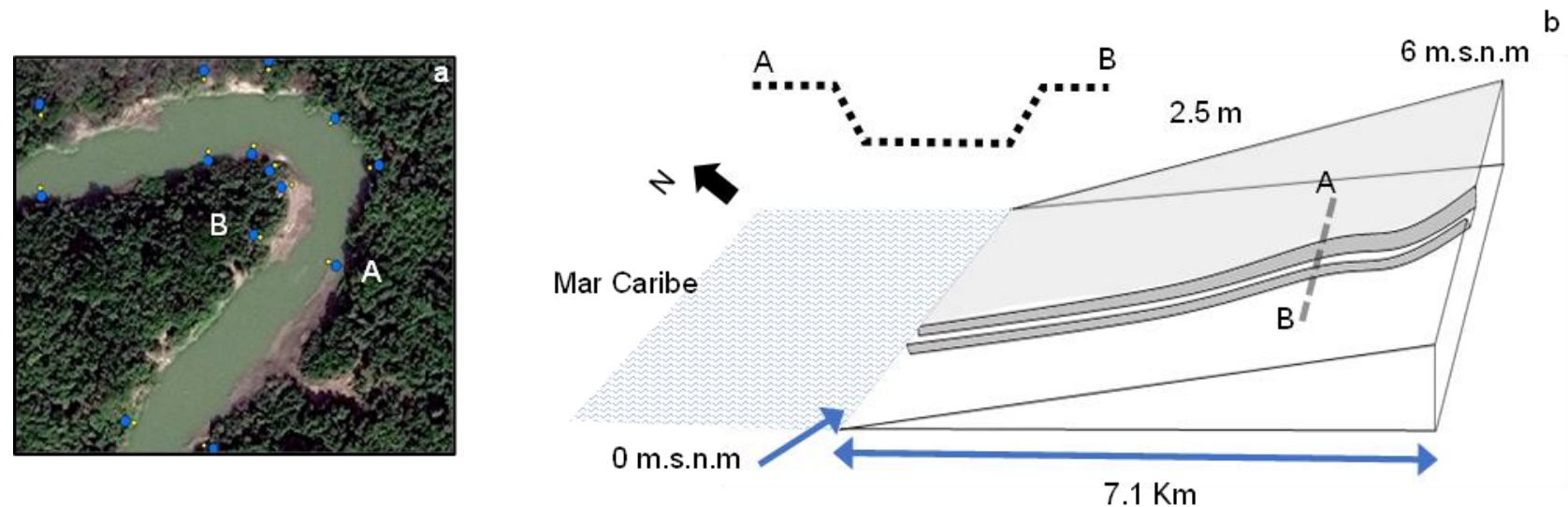

Fig. 3: Diseño del cauce del río. Ilustración visual de puntos en archivo SIG shapefile de las márgenes y fondo del cauce (a) y representación idealizada de canal trapezoidal para el cauce del rio Ranchería (b).

Generación del MDT preliminar. Los dos (2) archivos de puntos tipo (SHP) 2D para llanura y cauce fueron integrados en SIG para unificar la información del terreno. Con el archivo unificado, se procedió a generar una superficie tridimensional a partir de la nube de puntos mediante la creación de una red de triángulos irregulares (TIN) del terreno "con la función "Create TIN" en ArcScene ${ }^{\mathrm{TM}}$ Esri® (paso 3). Un primer esquema de ajuste 
iterativo fue establecido (paso 4), en el cual supervisadamente se hacían mejoras en la nube de puntos hasta obtener la mejor respuesta visualmente del ajuste del TIN con base a los datos iniciales, recorridos de campo y criterio del equipo investigador. Finalmente, el MDT preliminar se generó a partir de la rasterización del TIN con la herramienta "TIN to Raster" en ArcMap ${ }^{\mathrm{TM}}$ Esri® (paso 5).

Simulaciones en IBER con MDT preliminar. La finalidad de esta etapa fue verificar fallos en el MDT preliminar directamente en IBER (paso 6 y 7). Para esto no fue necesario llegar a la parametrización definitiva en IBER con las condiciones reales de inundación. Bastaba con asignar al azar un dato de caudal de los más altos registrados en la ola invernal 2010-2011 para evaluar la respuesta del MDT y su explicación al flujo en el cauce y llanura. Las salidas de simulación eran evaluadas visualmente hasta obtener repuestas aceptables para continuar con la preparación de datos necesarios para la parametrización final del modelo.

Obtención del MDT definitivo. Finalmente, para llegar a la obtención de un MDT definitivo se realizó un último esquema de ajuste iterativo, en el cual de forma supervisada (manualmente), se hacían mejoras en la nube de puntos primaria con el fin de evaluar visualmente la respuesta de las simulaciones preliminares en IBER (paso 8). Este proceso fue realizado cuantas veces era necesario hasta corregir en el cauce y llanura todas las anomalías, inconsistencias y errores mediante múltiples iteraciones. Así se obtuvo el mejor diseño representado en el MDT definitivo que visualmente representó un flujo coherente (paso 9). Este MDT fue usado para la parametrización de IBER 2D y así realizar las simulaciones definitivas de la alternativa base (Alternativa_0) de referencia y de protección contra inundaciones (Alternativa_1).

\section{Simulación de alternativas con el modelo IBER 2D}

Para llegar a la simulación de alternativas una vez obtenido el MDT definitivo, se continua con el procedimiento usual en IBER descrito en la figura $2 \mathrm{~b}$ con los siguientes pasos: parametrización del modelo IBER (Pasos 10), la definición de las alternativas de simulación (Paso 12), ejecución de las simulaciones de estabilidad (Paso 13), simulación de Alternativa_0 (Paso 14) y simulación Alternativa_1 (Paso 15). Por último, la exportación de las salidas ráster de la simulación para análisis en SIG (Paso 16).

Parametrización del modelo. El paso 10 es la preparación de los datos hidrológicos históricos y definición de las condiciones hidrodinámicas y mallado de la zona como sigue: (i) establecimiento de condición inicial del rio; (ii) identificación de crecidas; (iii) condiciones de contorno-entrada y salida; (iv) malla de cálculo y tamaño de elementos; y (v) supuestos y limitaciones de la modelación tal como se describe a continuación.

La condición inicial fue definida a partir de los datos hidrográficos existentes en una de las cinco estaciones hidrométricas oficiales (http://www.ideam.gov.co/solicitud-de-informacion). Esta se encuentra en la cuenca del río Ranchería de tipo Automática que cuenta con registros diarios desde el año 2007, ubicada en el corregimiento de Aremasahin identificada con el Código 15067180 (Figura 1). Es la estación más cercana al delta y al límite superior del área de estudio. La condición inicial en IBER está representada por un hidrograma. Esta condición se define por el caudal total en función del tiempo, para ello fue necesario encontrar el caudal permanente para el día 17/09/2011 y a partir de aquí construir el hidrograma (17/09/2011 - 24/09/2011). Lo anterior debido a la fecha del evento histórico de máxima precipitación en la zona urbana de la ciudad de Riohacha (18/09/2011). Al no existir registros de caudales a paso horario, se restringe la simulación a una frecuencia diaria con las implicaciones de menor detalle. Importante aclarar que esta investigación fue desarrollada en el periodo noviembre de 2015 a abril de 2016.

Para la Identificación de crecidas se realizó una prueba de distribución (Log Normal, Log Norma I, Gumbell, Pearson III y Log Pearson III) a los datos de caudal disponibles y se obtuvo la de mejor ajuste y con ella los caudales a varios tiempos de retorno $\left(T_{R}\right)$. Por otro lado, se determinó la capacidad del cauce en 5 secciones transversales entre ellas la condición de entrada (Puente punto 4 Figura 1), usando el programa HCANALES (http://maximovillon.blogspot.com.es/p/hcanales.html) a diferentes coeficientes de fricción " $n$ " de Manning considerando valores típicos para canales naturales. Estos caudales fueron comparados con los obtenidos en la mejor distribución de probabilidad determinando así el "n" más adecuado para cada $T_{R}$. El caudal de crecida en el puente fue el promedio entre caudales. Para la planicie de inundación los valores de "n" se han encontrado entre 0,05 a 0,07 (Pérez et al, 2010), por tanto, se decidió tomar el promedio de este intervalo $(0,6)$.

Las condiciones de contorno fueron definidas dentro el dominio de cálculo dibujado en SIG en una capa (shapefile) tipo polígono para la llanura de inundación y para el cauce del rio (Figura 4a). Posteriormente la información geométrica y MDT definitivo es importada en IBER para la asignación de condiciones hidrodinámicas específicas de la zona de estudio. La condición de salida de "tipo cota" que corresponde al máximo nivel presentado por la mar definida como régimen "Subcritico" fue impuesta en la línea de costa sobre el Mar Caribe (Figura 4b). Esta cota fue tomada del proyecto MODCEL fijada en 1 m.s.n.m (CREACUA, 
2015). La entrada corresponde al puente sobre la vía a Maicao, próxima a la estación automática hidrográfica (Figura 4c). Se asignó el hidrograma anteriormente construido en el contorno aguas arriba con un régimen de flujo "Crítico/Subcritico" usando la opción "caudal total".

Se creó una malla de cálculo irregular en todo el dominio, se asignaron los tamaños de elementos a generar (Cauce/llanura) de acuerdo al nivel de detalle requerido en cada una de las zonas tal como se muestra en la Figura 4d. En la llanura izquierda (en dirección aguas abajo), el detalle requerido era mayor por ser la limítrofe con la zona urbana y donde se disponía de mediciones de profundidad para la validación. El tamaño de los elementos de la malla en el río fue de $15 \mathrm{~m}$, para el lado izquierdo $50 \mathrm{~m}$ y $100 \mathrm{~m}$ para la llanura derecha (Figura 4e). Generada la malla de cálculo, se asignó el MDT definitivo.
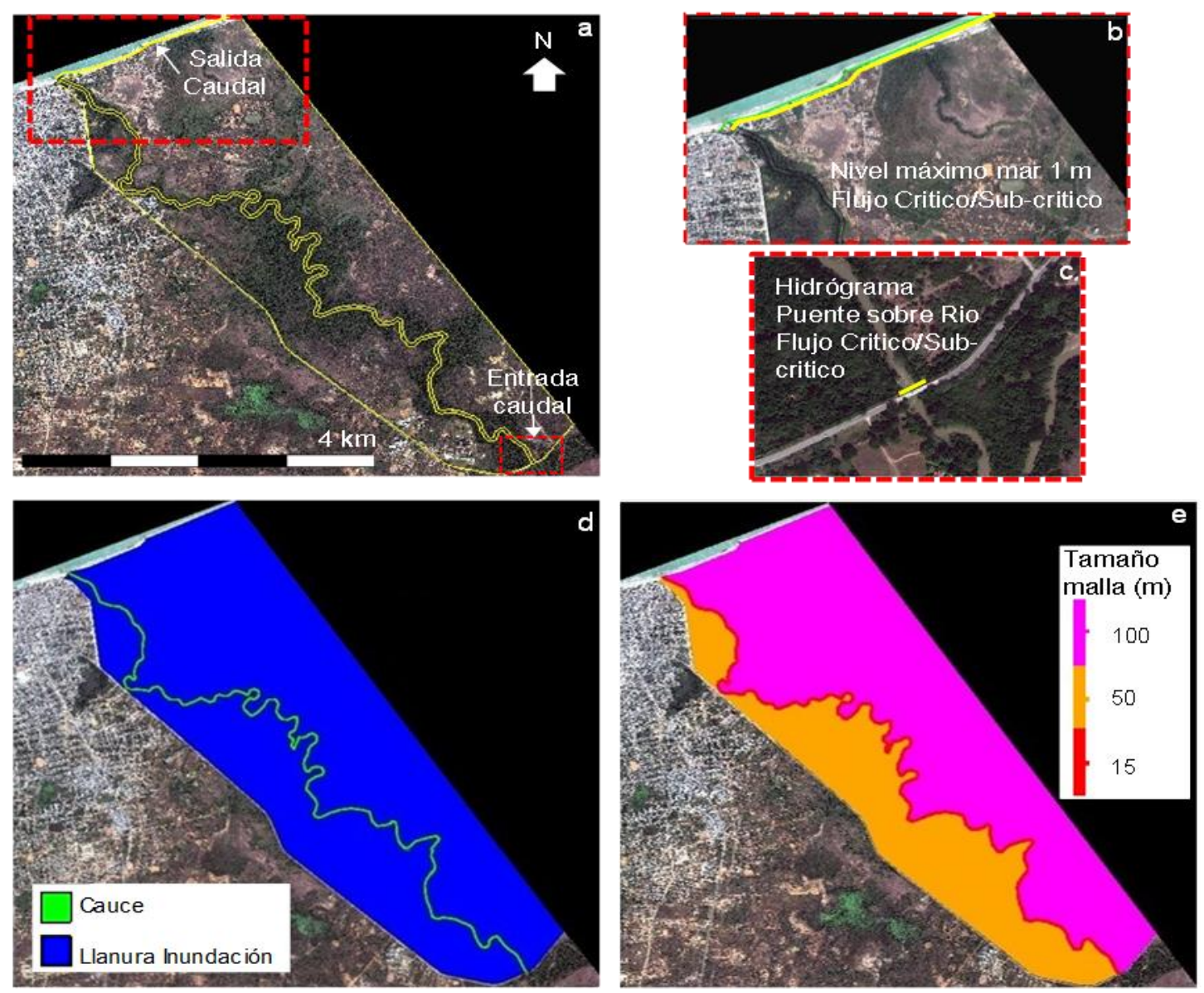

Fig. 4: Productos necesarios para la modelación. Dominio geométrico de cálculo (a), condición de salida (b), condición de entrada (c), asignación de rugosidad (d) y tamaños de malla para cauce y llanuras de inundación (e).

Los supuestos y limitaciones de la modelación son: (i) Se considera que el caudal registrado en la estación Aremasahin ubicada a $17 \mathrm{~km}$ en línea recta aguas arriba del dominio de cálculo es el mismo a la entrada del modelo y por tanto sin considerar aportes por escorrentías, tributario (La Quebrada) o extracciones adicionales. Los registros disponibles son de paso diario, no teniendo una frecuencia adecuada para modelar inundaciones; (ii) los datos topográficos (ASTER y Google Earth ${ }^{\mathrm{TM}}$ ) tienden a sobreestimaciones de la elevación por vegetación densa, lo que afecta en la producción del MDT definitivo y al final en las simulaciones pretendidas; (iii) por la amplitud de la zona y escasez de datos no se consideraron los otros dos brazos del río (Figura 1), lo que hace difícil saber si parte de la zona inundada al final de la desembocadura (barrio Villa Fátima) pudiese ocurrir por influencia del brazo Calancala (brazo 2); (iv) el cauce se dibujó con una geometría trapezoidal con profundidades y pendientes constantes en todo su recorrido; (v) el MDT tiene limitaciones en explicar la morfología del terreno en algunas zonas con cobertura boscosa impidiendo conocer las elevaciones en estos sitios.

Definición de Alternativas de simulación. Dos casos fueron planteados (Paso 12): Alternativa_0, es la simulación del evento base de referencia del 18/09/2011 que generó la máxima inundación en la ciudad de Riohacha en el periodo de la Ola invernal 2010-2011 (Nardini y Miguez, 2016). El interés de esta alternativa es determinar si el rio Ranchería influyó en la inundación urbana de la fecha de referencia. La Alternativa_1, consiste en modificar la malla agregando un dique de $1 \mathrm{~m}$ de alto y $3 \mathrm{~m}$ de ancho para verificar la protección contra las inundaciones del rio a una comunidad vulnerable residente dentro del delta (Villa Fátima). 
Ejecución de simulaciones. Una simulación de estabilidad fue ejecutada para establecer las condiciones hidrodinámicas iniciales antes del evento de referencia (Paso 13). Para verificar la estabilidad se realizó una simulación inicial hasta cuando la cota y calado fueron estables (se aprovecha de la asintótica estabilidad de los sistemas naturales). Lograda la estabilidad del río y llanura de inundación, se asignó el Hidrograma, partiendo desde el instante de tiempo en que se encontraron condiciones estacionarias, iniciando con la Alternativa_0 (Paso 14). Creada en la malla de cálculo y la recreación del dique, se procedió a simular la Alternativa_1 (Paso 15). Las salidas de simulación son exportadas en profundidades (metros) tipo formato ráster para ser analizadas en un SIG (Paso 16). Los resultados de simulación fueron validados comparando con las profundidades alcanzados en 3 puntos definidos en condición de frontera para el casco urbano de Riohacha, que coincide con los límites del delta y definidos en el proyecto MODCEL (CREACUA, 2015), esta frontera se definió físicamente como el nivel del agua que se alcanzó sobre la vía que conduce a Maicao y la carrera 1 (Figura 1, avenida circunvalar) por donde el río puede drenar hacia la ciudad cuando los niveles sobrepasan la capacidad del cauce. Esta frontera periurbana está sujeta a inundaciones, sea por las lluvias sobre la ciudad o crecidas del río.

\section{RESULTADOS Y DISCUSIÓN}

MDT definitivo para simulaciones. La nube de puntos topográficos integrada de cauce y llanura obtenida alcanza un total de 3110 puntos, distinguidos en color negro para la llanura de inundación y rojos para el cauce (Figura 5a). A partir de la nube de puntos obtenida, la red TIN interpolada se compone de 6196 triángulos con elevaciones que van desde los $-3 \mathrm{~m}$ del fondo del cauce hasta los $11 \mathrm{~m}$ del contorno aguas arriba (Figura 5b). El MDT definitivo en formato ráster (Figura 5c), obtenido a partir del TIN, se obtuvo con un tamaño de celda (resolución espacial) de 23,4 m (248 columnas x 248 filas). Por último, el MDT es importado en IBER para la creación de la malla de cálculo computacional con 21601 nodos y 36704 elementos en donde el código IBER 2D resuelve las ecuaciones hidrodinámicas de flujo bidimensional (Figura 5d).

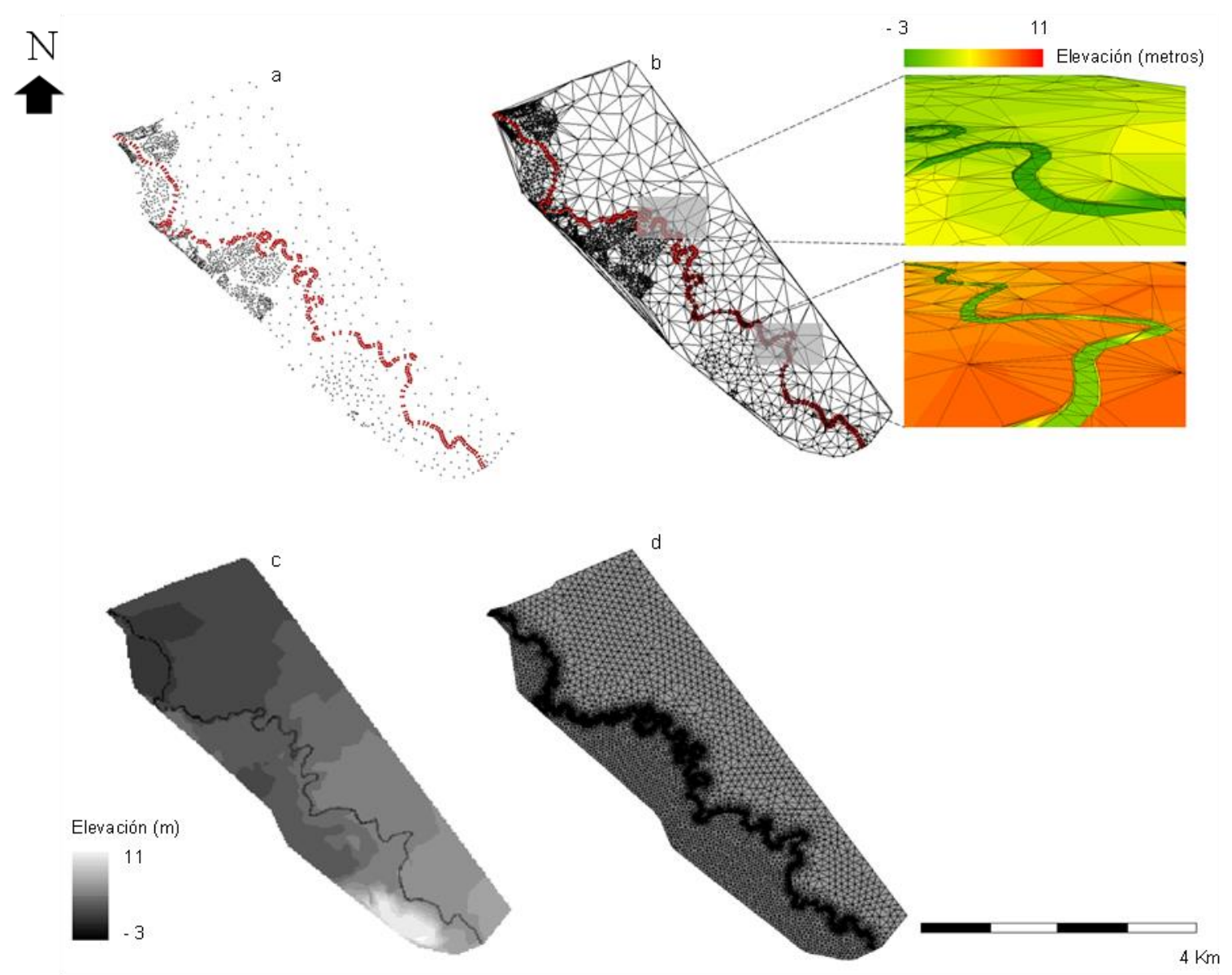

Fig. 5. Resultados de Información topográfica solventada con escasez de datos. Nube de puntos topográficos para llanura (negros) y río (rojos) (a), superficie interpolada TIN y acercamiento (b), vista en planta del MDT definitivo (c), y malla de cálculo obtenida en IBER (d).

Se buscó que la densidad promedio en la adquisición de puntos topográficos fuera adecuada $(\approx 3$ punto/ hectárea), con el fin de generar a partir del TIN y su posterior rasterización, una completa representación de la topografía. Lo anterior sin caer en una sobresaturación de detalle como recomiendan de Moel et al. (2015), que sugieren un establecimiento claro de los objetivos de evaluación del riesgo de inundación para determinar 
la escala de trabajo (global, nacional o regional o micro), y recursos óptimos para la modelación. Hay que evitar una generalización excesiva que pueda obviar elementos morfológicos del terreno propios de la llanura de inundación. Sin embargo, para el diseño del cauce, fue necesario una densificación alta de puntos para la representación de su geometría y en zonas próximas a los asentamientos y viviendas. Durante la parametrización en IBER 2D, se observó que un tamaño de celda del MDT mayor ( $>23,4 \mathrm{~m}$ ) y alta densidad de puntos, ralentiza más los tiempos de cálculo para la obtención de las simulaciones. Por tanto, la resolución espacial de 23,4 m fue la óptima, es decir, minimiza los tiempos de cálculo y máxima los recursos computacionales disponibles del estudio.

Por otro lado, el tratamiento de los datos en un SIG permitió el mejoramiento continuo del modelo topográfico con base al criterio de campo de los investigadores sobre aspectos geométricos tanto del cauce del rio como su llanura de inundación. La topografía plana de la zona, imprimió un mayor grado de dificultad para recrear las pendientes sutiles, pero necesarias para la generación dinámica del flujo. En esta investigación se hace un ejercicio de modelación del mundo real, considerando que el nivel de detalle y escala de trabajo lo definen los objetivos del fenómeno a simular. Lo anterior es crucial para la optimización de recursos computacionales y tiempos en la esquematización de la modelación. En el análisis de riesgo de inundación Apel et al. (2009) y Teng et al. (2017) concluyen que, a mayor nivel de detalle en la realidad a modelar, resulta una mayor complejidad, repercutiendo en una mayor demanda de recursos y tiempos de cómputo. La metodología aquí propuesta, aunque medianamente económica en tiempos de procesamiento computacional (Procesador Intel i5; 8 GB RAM), permitió una generalización de la morfología de la llanura y cauce sin profundizar significativamente en detalles. EI MDT fue adecuado para la escala de trabajo y condiciones de relieve del área de estudio, se podría decir que es equivalente a los MDT obtenidos por metodologías de "quemado de cauce" a escala de macrocuenca (Lindsay 2016). De esta forma se evadió posibles adquisiciones onerosas de datos geomáticos o campañas topográficas lo cual hubiera salido de los alcances y capacidades del presente estudio.

Simulación de alternativas. A continuación, se presentan los resultados de la parametrización del modelo (condición inicial-hidrograma e identificación de crecidas), la simulación de la estabilidad y de las dos alternativas realizadas en el presente estudio.

Condición inicial-Hidrograma. Analizando los datos de caudal registrados en la estación, se observaron valores similares del 10/09/2017 al 17/09/2011, el caudal promedio de estos días fue considerado como el caudal permanente $\left(58,51 \mathrm{~m}^{3} / \mathrm{s}\right)$. Este se incrementa hasta el $24 / 09 / 2011$, definiéndose así el periodo del hidrograma (17/09/2011 - 24/09/2011). En el presente caso, pese a contar con datos directos cerca al área de estudio, fueron detectados datos anómalos, días sin registros y al final una ventana de observación relativamente corta, restringiendo así un análisis histórico hidrológico riguroso, por lo menos de 20 años. Lo anterior, de cara a un escenario adverso de cambio climático según Guillot, et al. (2017), hace más justificable el desarrollo de tecnologías adecuadas para captura de información hidrológica de soporte al diseño de planes de adaptación a inundaciones. De esta forma se buscaría disminuir la incertidumbre en las estimaciones de los modelos hidrológicos y generación de mapas de inundación.

Identificación de crecidas. De las distribuciones utilizadas para el análisis de frecuencia de los caudales, la mejor fue la Log Pearson III con la cual se analizaron los caudales a tiempos de retorno ( $\left.T_{R}\right)$ de 2, 5, 10, 25, 50 y 100 años. El caudal de bancas llenas (capacidad hidráulica) en la sección de entrada se determinó con el coeficiente " $n$ " de Manning de 0,025; 0,030; 0,035 y 0,040. Los caudales más similares fueron los obtenidos con un $T_{R}$ de 2 años $\left(145,56 \mathrm{~m}^{3} / \mathrm{s}\right)$ y " $\mathrm{n}$ " de 0,025 (121,25 m³/s). Se tomó entonces como umbral mínimo el promedio entre ellos resultando un caudal de $133,40 \mathrm{~m}^{3} / \mathrm{s}$. A partir de este valor se consideró que existen crecidas en esta sección. Para el año 2011 solo se identificaron crecidas en el mes de junio, por tanto, el primer hallazgo es que para el evento de referencia (18/09/2011) no hubo crecida del río Ranchería. Días antes del evento e incluso horas, estas inundaciones se originaron de la precipitación intensa sobre la ciudad transformada en escorrentía urbana. Las inundaciones por lluvias (pluviales) o crecidas (fluviales), pueden ocurrir de forma simultánea, lo que no se presentó en nuestro caso, siendo esta probabilidad conjunta de ocurrencia difícil de estimar (Apel et al., 2016), más sin embargo, este escenario latente se presume podría ocurrir y sería significativamente desastroso con respecto a los daños y pérdidas del evento en referencia.

Simulación de estabilidad. Esta simulación se realizó con el caudal permanente $\left(58,51 \mathrm{~m}^{3} / \mathrm{s}\right)$ que corresponde al tiempo cero del hidrograma. El tiempo estimado para esta simulación fue de $45000 \mathrm{~s}$, en la cual se verificó la evolución del calado y la cota. Esta prueba demostró que la estabilidad en el cauce se logró antes de los $45000 \mathrm{~s}$ pero no en la planicie de inundación. El desbordamiento del río fue observado muy cerca a la desembocadura del río, lo que se debió a la condición de borde de salida impuesta por el nivel medio del mar. Por lo anterior fue necesario continuar la simulación hasta $90000 \mathrm{~s}$.

Simulación de la Alternativa_0. Esta se inició a los $90000 \mathrm{~s}$, el caudal permanente $\left(58,51 \mathrm{~m}^{3} / \mathrm{s}\right)$ fue necesario incluirlo para refrescar el proceso. En la Figura 6 se aprecian los resultados gráficos tipo ráster de profundidades de inundación alcanzadas a varios tiempos de paso. Como se puede observar, el 
desbordamiento se presentó muy cerca a la desembocadura, en ambos márgenes, llegando hasta la comunidad aledaña a Villa Fátima. Lo anterior se justifica porque el caudal simulado no excedía la capacidad hidráulica, es decir no debe existir desbordamiento en otras secciones aguas arriba. El desbordamiento en la parte final se debió a la influencia de la cota del mar (1 m.s.n.m), lo que ejerció un efecto barrera impidiendo la descarga del río.

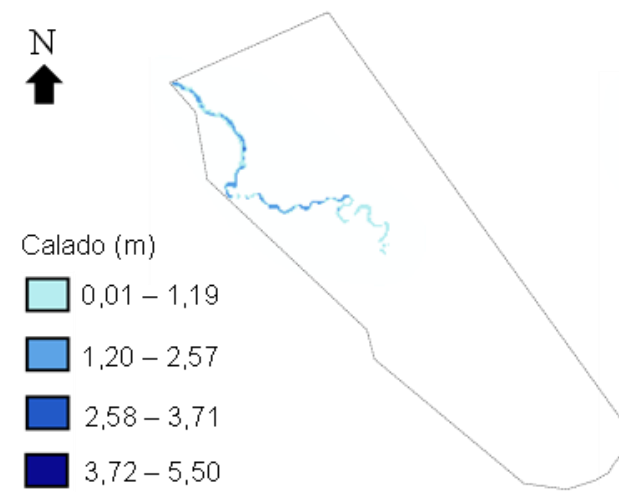

$0 \mathrm{~s}$

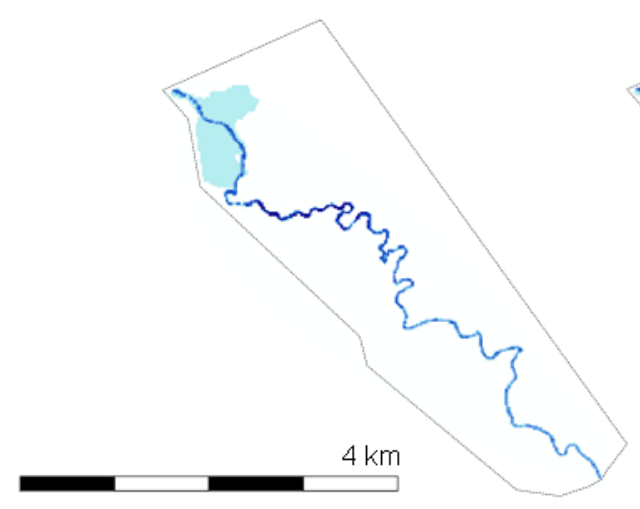

$21600 \mathrm{~s}$

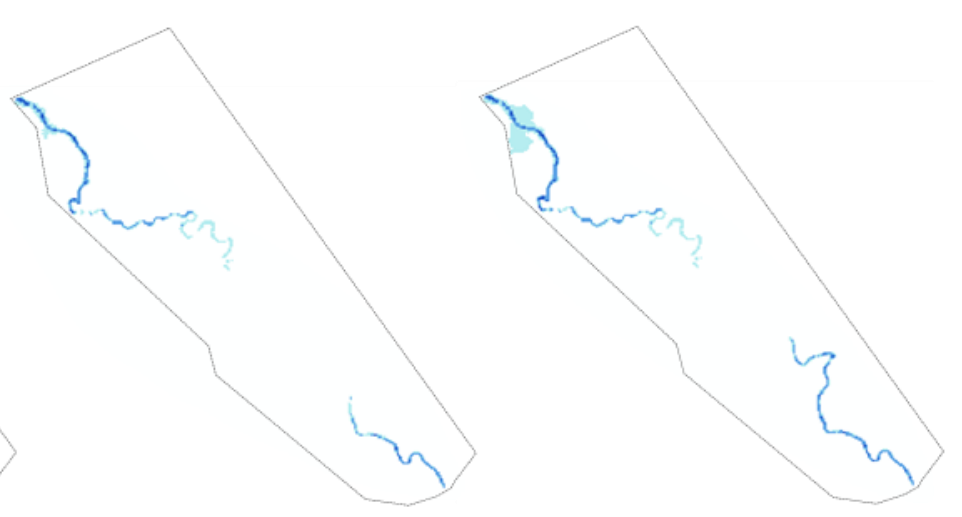

$1550 \mathrm{~s}$

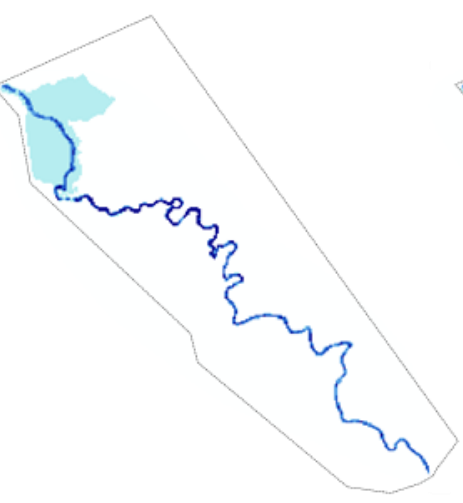

$350000 \mathrm{~s}$
$4300 \mathrm{~s}$

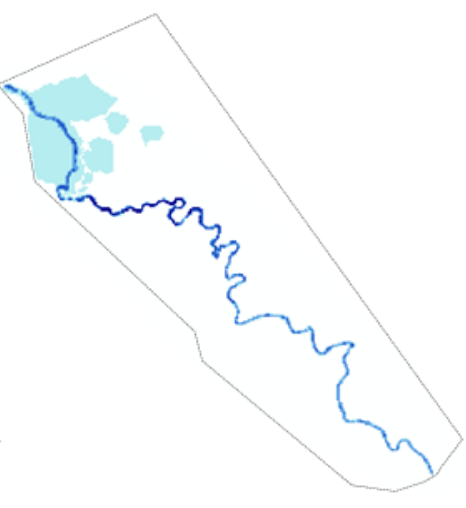

$604800 \mathrm{~s}$

Fig. 6: Salidas de simulación Alternativa_0 tipo ráster del calado (profundidad) en función del tiempo.

En la Figura 6 se puede observar que el calado del agua en el cauce empieza a aumentar gradualmente con el paso del tiempo siguiendo la forma del Hidrograma, llegando a un máximo alrededor de los $350000 \mathrm{~s}$ que coincide con el tiempo en que se registra el caudal pico. Lo anterior permitió evidenciar el efecto amortiguador del delta, es decir a medida que el río va desbordando la inundación se extendiendo. Por tanto, cuando los calados llegaron a la condición de estabilidad, se mantuvieron durante el tiempo de simulación, cambiando el área inundada, pero siempre cerca a la desembocadura.

Con respecto a la validación de los resultados, se pudo determinar que de los tres puntos ubicados en la frontera del área de estudio (CREACUA, 2015), solo se inundó el más cercano a la desembocadura donde la cota media en el proyecto MODCEL alcanzó 1,2 m.s.n.m y la simulada IBER de 1,0 m.s.n.m. Los dos puntos ubicados sobre la vía a Maicao (los box culvert la Vía y Casa Vivero) no fueron inundados a pesar que presentaron inundación en la ola invernal 2010-2011. Lo que se puede explicar porque en el proyecto MODCEL se demostró que el agua durante el evento de referencia fluyó del área urbana hacia el delta y no lo contrario. En otro evento de la misma ola invernal la zona en mención pudo ser inundada por el río, pero no es el caso en la lluvia tomada como referencia en nuestro estudio. Circunstancias diferentes pueden ocurrir en algún momento que coincida la inundación pluvial con la fluvial, como lo estudiado en el rio Can Tho de la ciudad de Vietnam (Apel et al., 2016), donde determinaron que el cálculo de los mapas de inundación y su probabilidad conjunta de ocurrencia está acompañado de considerable incertidumbre dada la variabilidad natural de estos eventos de inundación y la misma calidad de los datos y modelos hidrológicos empleados. Esta ausencia de información es similar a nuestro contexto, por tanto, se sugiere una evaluación de riesgos de inundación determinística con datos de insumo de eventos históricos más extremos apoyados en nuevas plataformas de teledetección para la confección de modelos empíricos de inundación como propone Malinowski et al. (2017) y Teng et al. (2017).

Una nueva e interesante ventana se abre en esta década con la información puntual de campo una vez ocurrido en evento extremo. Información de sensores espaciales de teledetección de agua por microondas y 
la irrupción de nuevas tecnologías emergentes de micro plataformas aéreas como las aeronaves remotamente pilotadas RPAs. La combinación de observaciones en tierra, satelital y RPAs ofrecen una nueva ventana para la complementación entre modelos de inundación 2D y empíricos. Pruebas recientes con los RPAs en la zona de estudio confirman el comportamiento de la escorrentía revelado por las salidas de simulación, al menos en las proximidades con la línea de costa. Estas tecnologías motivan a desarrollar investigaciones orientadas a evaluar los riesgos por inundaciones a partir de modelos empíricos y numéricos (Coveney y Roberts, 2017).

Simulación de la Alternativa_1 (Intervención). Los resultados son similares a la alternativa_0, pero se logra con el dique evitar artificialmente la inundación en la zona objeto de protección (Comunidad Villa Fátima). El efecto observado es en un ligero aumento del calado en la llanura contraria (limítrofe con la zona urbana). En la Figura 7 se puede observar una comparación de la inundación a los 600000 s para la Alternativaa_0 (Figura 7a) y Alternativa_1 (Figura 7b). Un efecto observado fue la propagación de la inundación por la zona desprotegida antes del punto de inicio del dique, este resultado pone de manifiesto que este tipo de obras a pesar de proteger y dar la sensación de seguridad aumenta el riesgo por posibles rupturas o fallas por una crecida de mayor magnitud.

Por otro lado, al aumentar el nivel del agua en el margen derecho se incrementaría el riesgo de inundaciones en la ciudad (margen izquierdo). Esta expansión lateral indeseada del rio puede magnificarse por todo tipo de barrera que afecta la descarga al mar. Esto fue observado luego de una creciente repentina (27/10/2015) al encontrarse la desembocadura del río Ranchería cerrada por una barrera de sedimentación debido al impacto de obras de protección tipo espigones construidas a lo largo de la línea de costa. En estas condiciones, el río fluyó hacia la ciudad por los box coulvert al no conseguir el natural descargue al mar, produciendo inundaciones considerables.

Es importe señalar que un dique es una obra que requiere mantenimiento para que cumpla su función en el tiempo. Al proteger artificialmente a la comunidad, el riesgo de inundación es mayor, especialmente por la gran probabilidad que la obra falle (Miller et al., 2015). La obra tipo "dura" o convencional de protección a la comunidad, incentivarían más el urbanismo descontrolado alrededor de la protección espuria del dique, aumentando la vulnerabilidad de las viviendas expuestas a la amenaza latente de inundación con una mayor liberación de energía de colapsar el dique. Para un evento de mayor magnitud al de referencia, la comunidad podría experimentar fuertes inundaciones a pesar la presencia de la obra, aun por falla estructural o simple rebose. El material constructivo del dique podría jugar un papel importante en la reducción del riesgo, si por el contrario es reemplazado por una obra "blanda" compuesto por vegetación y materiales convencionales como sugiere los nuevos enfoques de adaptación basado en ecosistemas (Spalding et al., 2014).

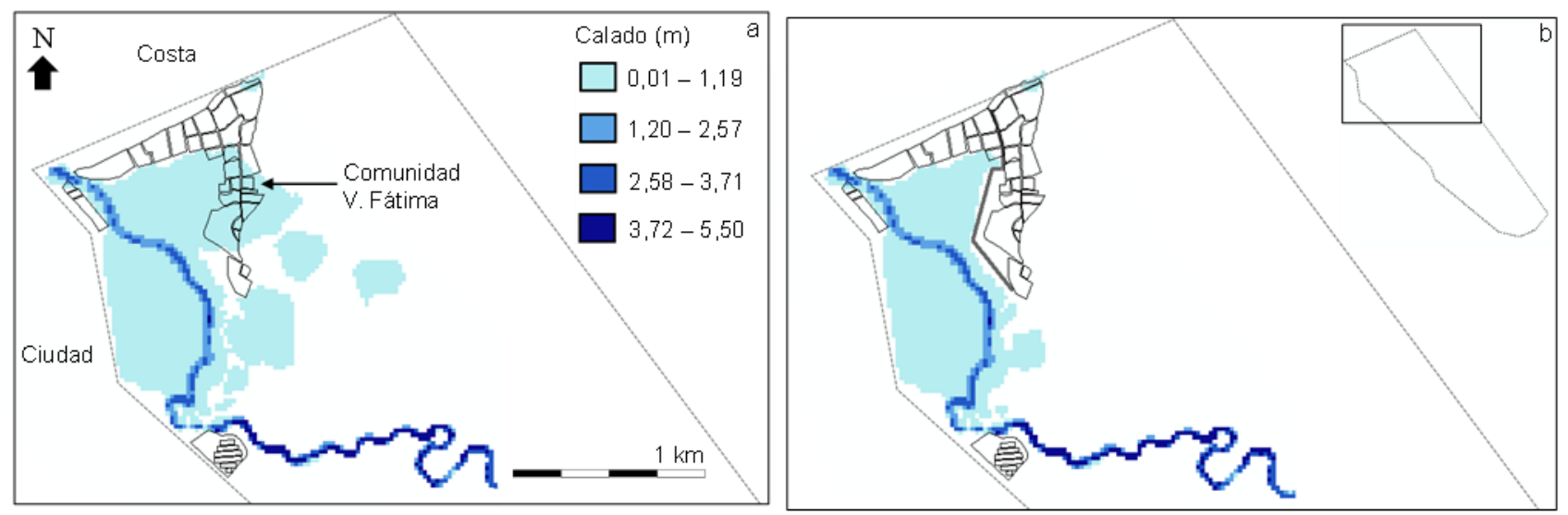

Fig. 7. Salidas de simulación para el instante 600000 s. Alternativa_0 (a) y alternativa_1 (b).

Estas nuevas tendencias ingenieriles buscan una integración con la dinámica local mientras aumenta el valor ambiental, mostrando un bajo costo de inversión y mantenimiento en comparación a obras de protección convencionales en zonas costeras (Reguero et al., 2017). Este ejercicio de simulación de la amenaza y daños, impactaría en generar conciencia de preparación en nuevos enfoques integrados con el entorno natural del delta ante la ocurrencia del escenario local más adverso de inundación: crecida del río Ranchería, con aumento de la marea o nivel medio del mar por el CC y las inundaciones de la escorrentía urbana.

\section{CONCLUSIONES}

A partir de los resultados obtenidos, se pueden plantear las siguientes conclusiones principales: 1.- La cobertura mundial de topografía de Google Earth ${ }^{\mathrm{TM}}$, el uso de herramientas libres como IBER y accesibilidad de los SIG, permitieron superar la escasez de datos topográficos y poder realizar la modelación 2D de 
inundaciones fluviales en una zona deltaica. Un producto clave fue el MDT que integra de forma idealizada la geometría del cauce y la morfología de la llanura de inundación del río Ranchería; 2.- Durante el evento de referencia (alternativa_0) no hubo crecida del rio Ranchería, es decir no existió coincidencia con la inundación pluvial, por tanto, el desbordamiento solo ocurrió en la parte final del río ocasionada por la barrera impuesta por la cota del mar; 3.- La alternativa_1, ofrece una protección artificial a la comunidad objeto de protección (Villa Fátima), pero trasladando la inundación ligeramente hacia la ciudad. Esto aumentaría el riesgo de inundación en temporadas de crecidas sugiriendo un nuevo enfoque de intervención ingenieril basado en ecosistemas; y 4.- Esta metodología es una opción accesible y viable de soporte a la modelación de inundaciones $2 \mathrm{D}$ en regiones donde la información topográfica de partida es una limitante. Lo que se convierte en una herramienta metodológica para que las instituciones competentes tomen decisiones que permitan gestionar el riesgo de inundación de forma acertada y con enfoques ingenieriles.

\section{AGRADECIMIENTOS}

A la Universidad de La Guajira por el apoyo económico, al Instituto de Hidrología, Meteorología y Estudios Ambientales (IDEAM) de Colombia por la información suministrada, a la fundación CREACUA por la información del proyecto MODCEL, A los ing. Andrea Nardini por sus asesorías, Yair Movil Fuentes por el apoyo topográfico y Yesenia Zuñiga Mendoza por sus invaluables aportes en la edición del manuscrito.

\section{REFERENCIAS}

Apel, H., G.T. Aronica, H. Kreibich y A.H. Thieken, Flood Risk Analyses-how Detailed do we Need to be? doi: http://doi.org/10.1007/s11069-008-9277-8, Natural Hazards, 49 (1), 79-98 (2009)

Apel, H., O.M. Trepat, N.N. Hung, B. Merz y N.V. Dung, Combined Fluvial and Pluvial Urban Flood Hazard Analysis: Concept Development and Application to can tho City, Mekong Delta, Vietnam, doi: https://doi.org/10.5194/nhess-16-9412016, Natural Hazards and Earth System Sciences, 16(4) 941-961 (2016)

Álvarez, M., J. Puertas, E. Peña y M. Bermúdez, Two-Dimensional Dam-Break Flood Analysis in data-Scarce Regions: the Case Study of Chipembe Dam, Mozambique, doi: https://doi.org/10.3390/w9060432, Water, 9(6), 432 (2017)

Bedoya, V.H. y J.M. López-Lezama, Modelo para el Control de Inundaciones durante el Fenómeno de "La Niña" Utilizando un embalse Hidroeléctrico, doi: https://doi.org/10.4067/S0718-07642015000200011, Inf. Tecnol. 26(2), 89-100 (2015)

Bodoque, J.M., M. Amérigo, A. Díez-Herrero, J.A. García, B. Cortés, J.A. Ballesteros-Cánovas y J. Olcina, Improvement of Resilience of Urban Areas by Integrating Social Perception in Flash-Flood Risk Management, doi: http://doi.org/10.1016/j.jhydrol.2016.02.005, Journal of Hydrology, 541, 665-676 (2016)

Bonasia, R., O.S. Areu-Rangel, D. Tolentino, I. Mendoza-Sanchez, J. González-Cao y J. Klapp, Flooding Hazard Assessment at Tulancingo (Hidalgo, Mexico), doi: http://doi.org/doi:10.1111/jfr3.12312, Journal of Flood Risk Management, 11, 1-9 (2017)

Bladé, E., L. Cea, G. Corestein, E. Escolano, J. Puertas, E. Vázquez-Cendón, J. Dolz y A. Coll, Iber: Herramienta de Simulación Numérica del Flujo en Ríos, doi: http://doi.org/10.1016/j.rimni.2012.07.004, Rev. Int. Metod. numér, 30(1), 1$10(2014)$

Pérez, A., A. Dorta y A. Marrero, El Coeficiente de Manning y el Cambio de Precisión de los MDT en La Simulación Hidráulica, http://riha.cujae.edu.cu/index.php/riha/article/view/41/89; ISSN: 1815-591X, Rev. de Ingeniería Hidráulica y Ambiental, 31(3), 21-27 (2010)

Cai, W., G. Wang, A. Santoso, M. McPhaden, L. Wu, T.F. Jin, A. Timmermann, M. Collins, G. Vecchi, M. Lengaigne, H.M. England, D. Dommengel, H. Takahashi y E. Guilyardi, Increased Frequency of Extreme La Niña Events Under Greenhouse Warming, doi: http://doi.org/10.1038/nclimate2492, Nature Climate Change, 5(2), 132-137 (2015)

Castillo, C., R. Pérez y J.A. Gómez, Conceptual Model Of Check Dam Hydraulics for Gully Control: Efficiency, Optimal Spacing and Relation with Step-Pools, doi: https://doi.org/10.5194/hess-18-1705-2014, Hydrology and Earth System Sciences, 18(5), 1705-1721 (2014)

CREACUA- Centro de Recuperación de Ecosistemas Acuáticos. Informe del Proyecto: Adaptación Urbana Verde Frente a Inundaciones con el Soporte de la Modelación Matemática y del Software MODCEL en Riohacha, La Guajira, Colombia; Convenio de Cooperación № 9677-04-1047-2013; CREACUA, Riohacha, La Guajira-Colombia (2015)

Coveney, S. y K. Roberts, Lightweight UAV Digital Elevation Models and Orthoimagery for Environmental Applications: Data Accuracy Evaluation and Potential for river Flood Risk Modelling, doi: https://doi.org/10.1080/01431161.2017.1292074, International Journal of Remote Sensing, 38(8-10), 3159-3180 (2017)

de Moel, H., B. Jongman, H. Kreibich, B. Merz, E. Penning-Rowsell y P.J Ward, Flood risk assessments at different spatial scales, doi: https://doi.org/10.1007/s11027-015-9654-z, Mitigation and Adaptation Strategies for Global Change, 20(6), 865-890 (2015)

Franco, F.L., Respuestas y Propuestas ante el Riesgo de Inundación de las Ciudades Colombianas, https://goo.gl/wdHTPA; ISSN: 0121-4993, Revista de ingeniería de la Universidad de Los Andes, 31, 97-108 (2010) 
Guillot, J.D., C.A. Robles y J.D. Callejas, Adquisición de Señales Ambientales para un Sistema de Alerta Temprana, doi: http://dx.doi.org/10.4067/S0718-07642017000500007, Inf. Tecnol. 28(5), 45-54 (2017)

Hoffmann, E. y F. Winde, Generating High-Resolution Digital Elevation Models for Wetland Research using Google Earth ${ }^{\text {tm }}$ Imagery: an Example from South Africa, https://goo.gl/LVbVTv; ISSN: 1816-7950, Water SA, 36(1), 53-68 (2010)

Hoyos, N., J. Escobar, J.C. Restrepo, A.M. Arango y J.C. Ortiz, Impact of the 2010-2011 La Niña Phenomenon in Colombia, South America: the Human toll of an Extreme Wather Event., doi http://dx.doi.org/10.1016/j.apgeog.2012.11.018, Applied Geography, 39, 16-25 (2013)

Kundzewicz, Z.W., S. Kanae y otros quince autores, Flood Risk and Climate Change: Global and Regional Perspectives, http://dx.doi.org/10.1080/02626667.2013.857411, Hydrological Sciences Journal, 59(1), 1-28 (2014)

Lindsay, J.B., The Practice of DEM Stream Burning Revisited. Earth Surface Processes and Landforms, doi: http://dx.doi.org/10.1002/esp.3888, 41(5), 658-668 (2016)

Malinowski, R., G.B. Groom, G. Heckrath y W. Schwanghart, Do Remote Sensing Mapping Practices Adequately Address Localized Flooding? A Critical Overview, doi: https://doi.org/10.1007/s40362-017-0043-8, Springer Science Reviews, 117 (2017)

Musa, Z.N., I. Popescu. y A. Mynett, A Review of Applications of Satellite SAR, Optical, Altimetry and DEM data for Surface Water Modelling, Mapping and Parameter Estimation, doi: https://doi.org/10.5194/hessd-12-4857-2015, Hydrology and Earth System Sciences, 19(9), 3755-3769 (2015)

Miller, A., S.N. Jonkman y M. Van Ledden, Risk to Life due to Flooding in Post-Katrina New Orleans, doi: https://doi.org/10.5194/nhess-15-59-2015, Natural Hazards and Earth System Sciences, 15 (1), $59-73$ (2015)

Nardini, A. y M.G Miguez, An Integrated Plan to Sustainably Enable the City of Riohacha (Colombia) to Cope with Increasing Urban Flooding, while Improving Its Environmental Setting, doi: https://doi.org/10.3390/su8030198, Sustainability, 8(3), 198 (2016)

OMM-Organización Mundial Meteorológica, el Estado del Clima Mundial 2001-2010. Un decenio de Fenómenos Climáticos Extremos Informe Resumido, Ginebra, Suiza (2012)

Ruslin, N., M.R. Majid y A.H. Din, Google Earth's derived Digital Elevation Model: A Comparative Assessment with Aster and SRTM data, doi: https://doi.org/10.1088/1755-1315/18/1/012065, Actas del 8th International Symposium of the Digital Earth (ISDE8), IOP Conf. Series: Earth and Environmental Science, 18 conference 1), IOP Publishing, Kuching, Sarawak, Malasia, 23 a 26 de mayo de 2013 (2014)

Reguero, B.G., M.W. Beck, I.J. Losada y S. Narayan, Uniendo Ingeniería y Ecología: la Protección Costera Basada en Ecosistemas, doi: https://doi.org/10.1080/23863781.2017.1332824. Ribagua, 4(1), 41-58 (2017)

Shen, D., J. Wang, X. Cheng, Y. Rui y S. Ye, Integration of 2-D Hydraulic Model And High-Resolution Lidar-Derived Dem for Floodplain Flow Modeling, https://doi.org/10.5194/hess-19-3605-2015, Hydrology and Earth System Sciences, 19(8), 3605-3616 (2015)

Tedeschi, R.G. y M. Collins, The influence of ENSO on South American Precipitation: Simulation and Projection in CMIP5 Models, doi: https://doi.org/10.1002/joc.4919, International Journal of Climatology, 37(8), 3319-3339 (2016)

Teng, J., A.J. Jakeman, J. Vaze, B.F. Croke, D. Dutta y S. Kim, Flood Inundation Modelling: A review of Methods, Recent Advances and Uncertainty Analysis, doi: https://doi.org/10.1016/j.envsoft.2017.01.006, Environmental Modelling and Software, 90, 201-216 (2017)

Spalding, M.D., S. Ruffo, C. Lacambra, I. Meliane, L.Z Hale, C.C. Shepard, y M.W. Beck, The Role of Ecosystems in Coastal Protection: Adapting to Climate Change and Coastal Hazards, doi: https://doi.org/10.1016/j.ocecoaman.2013.09.007, Ocean \& Coastal Management, 90, 50-57 (2014)

Trenberth, K.E., Changes in Precipitation with Climate Change, doi: https://doi.org/110.3354/cr00953, Climate Research, 47(1-2), 123-138 (2011)

Yan, K., G.Di. Baldassarre, D.P. Solomatine y G.J.P. Schumann, A Review of Low-Cost Space-Borne data for Flood Modelling: Topography, Flood Extent and Water Level, doi https://doi.org/10.1002/hyp.10449, Hydrological Processes, 29 (15), 3368-3387 (2015) 
